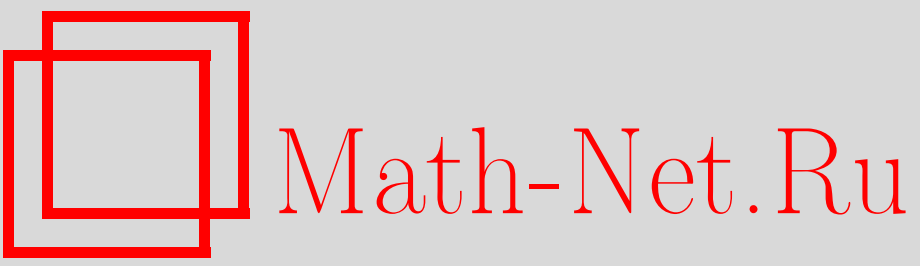

Л. Д. Пустыльников, Пространственно-временной хаос, критические явления и бифуркации решений бесконечномерных систем обыкновенных дифференциальных уравнений, ТМФ, 2002, том 133, номер 1, 36-53

DOI: https://doi.org/10.4213/tmf379

Использование Общероссийского математического портала Math-Net.Ru подразумевает, что вы прочитали и согласны с пользовательским соглашением

http://www.mathnet.ru/rus/agreement

Параметры загрузки:

IP: 35.173 .219 .12

26 апреля 2023 г., 15:07:48 
ТЕОРЕТИЧЕСКАЯ

И МАТЕМАТИЧЕСКАЯ

ФИЗИКА

Том 133, № 1

октябрь, 2002

(C) 2002 г.

Л. Д. Пустыльников*

\section{ПРОСТРАНСТВЕННО-ВРЕМЕННОЙ ХАОС, КРИТИЧЕСКИЕ ЯВЛЕНИЯ И БИФУРКАЦИИ РЕШЕНИЙ БЕСКОНЕЧНОМЕРНЫХ СИСТЕМ ОБЫКНОВЕННЫХ ДИФФЕРЕНЦИАЛЬНЫХ УРАВНЕНИЙ}

В работе изучаются бесконечномерные системы обыкновенных дифференциальных уравнений, имеющие применения в популярных и важных физических проблемах. Рассматривается возникновение бесконечномерного пространственно-временного хаоса: описываются бифуркации и критические явления в фазовом пространстве систем, дающие объяснения некоторых физических проблем.

Ключевые слова: хаос, бифуркация, устойчивость, бесконечномерная система уравнений, гиперболическая точка, сепаратриса.

\section{1. ВВЕДЕНИЕ}

Эта работа посвящена математически строгому исследованию критических явлений и устойчивого хаоса в неконсервативных физических системах, состоящих из очень большого (практически бесконечного) числа частиц. Типичный и очень популярный пример такой системы - куча песка, находящаяся под действием внешних сил. В этом примере действуюшие между частицами силы основаны на законе трения, и поэтому дифференциальные уравнения, описывающие их движение, содержат первые (а не вторые, как в консервативных системах классической механики) производные.

Опишем основные физические проблемы, относящиеся к рассматриваемой модели. Предположим, что песок находится под действием достаточно гладких внешних сил, зависящих от параметров: например, песок из внешнего источника сыплется на поверхность кучи. Форма кучи принципиально не меняется во все моменты времени, для которых масса песка меньше или равна, чем некоторое критическое значение, но если масса песка превосходит это значение, то происходит резкое изменение формы. Другими

\footnotetext{
${ }^{*}$ Институт прикладной математики им. М. В. Келдыша РАН, Москва, Россия. E-mail: pustylni@physik.uni-bielefeld.de
} 
словами, здесь мы наблюдаем бифуркацию или, согласно терминологии статистической физики, фазовый переход [1]. Далее, первоначальная форма кучи песка может быть очень сложной и хаотичной, но тем не менее устойчивой в том смысле, что при малом возмущении формы происходит ее восстановление. Подобные проблемы возникают во многих других весьма актуальных задачах, таких как сход лавин, образование фиордов, распространение пожаров и эпидемий, функционирование нейронных сетей, действие клеточных автоматов, явление сверхпроводимости, распределение галактик и т.д. [2]. В настояшее время применительно к этим системам вошел в употребление термин "сложность"; это подразумевает, что их поведение характеризуется высокой сложностью, связанной с очень большой размерностью фазового пространства систем описывающих их дифференциальных уравнений. Именно поэтому методы, основанные на сушествующих теориях бифуркаций и катастроф [3], в данном случае трудно применить. Методы же, основанные на теории фазовых переходов в классической статистической физике, нельзя использовать ввиду отсутствия естественной инвариантной меры.

В настояшей работе указанные проблемы изучаются для широкого класса систем, состояших из бесконечного числа частищ $P_{\vec{n}}$, занумерованных всеми $r$-мерными $(r \geqslant 1)$ векторами $\vec{n}=\left(n_{1}, \ldots, n_{r}\right) \in \mathbf{Z}^{r}$ с целыми координатами $n_{k}(k=1, \ldots, r)$, которые двигаются в $r$-мерном вешественном пространстве $\mathbf{R}^{r}$. Здесь $r$-натуральное число, а $\mathbf{Z}^{r}-$ $r$-мерная целочисленная решетка в $\mathbf{R}^{r}$. Мы предполагаем, что каждая частица $P_{\vec{n}}$ взаимодействует только с соседними частицами, расположенными вдоль каждого направления в $\mathbf{Z}^{r}$, и все частищы находятся во внешнем силовом поле, которое действует в направлении каждой координатной оси в $\mathbf{R}^{r}$. Точка $P_{\vec{n}}$ характеризуется $r$-мерным вектором $x_{\vec{n}}=\left(x_{\vec{n}}^{(1)}, \ldots, x_{\vec{n}}^{(r)}\right) \in \mathbf{R}^{r}$, а общий формальный потенциал взаимодействия $U$ имеет вид

$$
U=\sum_{\vec{n} \in \mathbf{Z}^{r}} \sum_{S=1}^{r} G^{(S)}\left(x_{\vec{n}}^{(S)}\right)+\chi \sum_{\left(\vec{n}^{\prime}, \vec{n}^{\prime \prime}\right) \in \Gamma}\left|x_{\vec{n}^{\prime}}-x_{\vec{n}^{\prime \prime}}-\vec{a}\right|^{2},
$$

где

$$
\begin{gathered}
\vec{n}^{\prime}=\left(n_{1}^{\prime}, \ldots, n_{r}^{\prime}\right) \in \mathbf{Z}^{r}, \\
\vec{n}^{\prime \prime}=\left(n_{1}^{\prime \prime}, \ldots, n_{r}^{\prime \prime}\right) \in \mathbf{Z}^{r}, \quad x_{\vec{n}^{\prime}}=\left(x_{\vec{n}^{\prime}}^{(1)}, \ldots, x_{\vec{n}^{\prime}}^{(r)}\right), \\
x_{\vec{n}^{\prime \prime}}=\left(x_{\vec{n}^{\prime \prime}}^{(1)}, \ldots, x_{\vec{n}^{\prime \prime}}^{(r)}\right) \in \mathbf{R}^{r}, \quad \vec{a}=\left(a^{(1)}, \ldots, a^{(r)}\right) \in \mathbf{R}^{r}, \\
\left|x_{\vec{n}^{\prime}}-x_{\vec{n}^{\prime \prime}}-\vec{a}\right|^{2}=\sum_{S=1}^{r}\left(x_{\vec{n}^{\prime}}^{(S)}-x_{\vec{n}^{\prime \prime}}^{(S)}-a^{(S)}\right)^{2} .
\end{gathered}
$$

Здесь $\Gamma$ - множество пар $r$-мерных векторов $\vec{n}^{\prime}, \vec{n}^{\prime \prime}$ с целыми координатами таких, что

$$
\sum_{S=1}^{r}\left|n_{S}^{\prime}-n_{S}^{\prime \prime}\right|=1, \quad \sum_{S=1}^{r}\left(n_{S}^{\prime}-n_{S}^{\prime \prime}\right)=1
$$

а сумма в правой части равенства (1.1) распространена на все пары $\left(\vec{n}^{\prime}, \vec{n}^{\prime \prime}\right) \in \Gamma$. Мы предполагаем, что $\vec{a}$ - заданный вектор, $\chi$ - константа, а $G^{(S)}(x)$ - бесконечно дифференцируемая функция на прямой $\mathbf{R}^{1}$, характеризуюшая внешнюю силу, действуюшую 
в направлении координаты $x_{\vec{n}}^{(S)}, S=1, \ldots, r$. Эта функция представима в виде

$$
G^{(S)}(x)=F^{(S)}(x)-\beta^{(S)} x
$$

где $F^{(S)}(x)$ - отличная от нуля функция, имеющая ограниченную по модулю производную, а $\beta^{(S)}$ - параметр. Если $\chi>0$, то с физической точки зрения система представляет собой $r$-мерную сеть пружинок, находяшуюся под действием внешнего поля с потенциалом $\sum_{\vec{n} \in \mathbf{Z}^{r}} \sum_{S=1}^{r} G^{(S)}\left(x_{\vec{n}}^{(S)}\right)$.

Рассмотрим теперь градиентную систему с бесконечным числом степеней свободы

$$
\frac{d x_{\vec{n}}}{d t}=-\frac{\partial U}{\partial x_{\vec{n}}}, \quad \vec{n} \in \mathbf{Z}^{r}
$$

где

$$
\frac{d x_{\vec{n}}}{d t}=\left(\frac{d x_{\vec{n}}^{(1)}}{d t}, \ldots, \frac{d x_{\vec{n}}^{(r)}}{d t}\right) \in \mathbf{R}^{r}, \quad \frac{\partial U}{\partial x_{\vec{n}}}=\left(\frac{\partial U}{\partial x_{\vec{n}}^{(1)}}, \ldots, \frac{\partial U}{\partial x_{\vec{n}}^{(r)}}\right) \in \mathbf{R}^{r}
$$

а для $S=1, \ldots, r$ величина $\partial U / \partial x_{\vec{n}}^{(S)}$ есть производная формального ряда $U$ относительно $x_{\vec{n}}^{(S)}$. В силу (1.1)-(1.3) система (1.4) принимает вид

$$
\frac{d x_{\vec{n}}^{(S)}}{d t}=-f^{(S)}\left(x_{\vec{n}}^{(S)}\right)+\beta^{(S)}+2 \chi\left(-2 r x_{\vec{n}}^{(S)}+\sum_{\vec{n}^{\prime} \in \Gamma_{\vec{n}}} x_{\vec{n}^{\prime}}^{(S)}\right), \quad \vec{n} \in \mathbf{Z}^{r},
$$

где $S=1, \ldots, r, \vec{n}=\left(n_{1}, \ldots, n_{r}\right) \in \mathbf{Z}^{r}, f^{(S)}\left(x_{\vec{n}}^{(S)}\right)=d F^{(S)}\left(x_{\vec{n}}^{(S)}\right) / d x, \Gamma_{\vec{n}}$ - множество всех векторов $\vec{n}^{\prime}=\left(n_{1}^{\prime}, \ldots, n_{r}^{\prime}\right) \in \mathbf{Z}^{r}$ таких, что $\sum_{S=1}^{r}\left|n_{S}-n_{S}^{\prime}\right|=1$, а $t$ - независимая переменная, характеризуюшая время. Согласно определению функции $F^{(S)}(x)$, в $(1.3)$ для всех $S=1, \ldots, r$ сушествуют константы $\gamma_{S}^{\prime}$ и $\gamma_{S}^{\prime \prime}$ такие, что

$$
\gamma_{S}^{\prime} \stackrel{\text { def }}{=} \min _{x \in \mathbf{R}^{1}} f^{(S)}(x) \leqslant \max _{x \in \mathbf{R}^{1}} f^{(S)}(x) \stackrel{\text { def }}{=} \gamma_{S}^{\prime \prime} .
$$

В данной работе проводится строгое качественное исследование бесконечномерной системы обыкновенных дифференциальных уравнений (1.5). Во втором разделе развивается общая теория системы (1.5), включающая конструкцию стационарных решений и фазовых пространств, теорию асимптотической устойчивости и построение бесконечномерных странных аттракторов. В разделе 3 изучаются некоторые семейства решений этой системы и их бифуркации относительно изменений параметров и начальных данных.

Отметим следуюшее обстоятельство. Предположим, что функции $f^{(S)}(x)$ $(S=1, \ldots, r)$, входяшие в систему $(1.5)$, имеют период 1 по $x$. Тогда, если векторы $x_{\vec{n}}(t)=\left(x_{\vec{n}}^{(1)}(t), \ldots, x_{\vec{n}}^{(r)}(t)\right), \vec{n} \in \mathbf{Z}^{r}$, удовлетворяют системе $(1.5)$, то векторы $y_{\vec{n}}(t)=$ $x_{\vec{n}}(t)+\vec{n}, \vec{n} \in \mathbf{Z}^{r}$, также удовлетворяют этой системе, и для них справедливы все основные утверждения этой работы. В частности, выводы о критических явлениях, бифуркациях и хаосе, примененные к векторам $y_{\vec{n}}(t)$, позволяют дать объяснения тем физическим явлениям, о которых говорилось в начале введения. 
Настоящую статью в известной степени можно считать обобшением работы [4], в которой изучался весьма частный одномерный случай системы (1.5) при $r=1, \chi=1 / 2$, $f^{(1)}(x)=b \sin x$.

Так как при разных значениях $S$ уравнения, входящие в систему (1.5), не зависят друг от друга, а вид их один и тот же, то в дальнейшем под системой (1.5) мы будем понимать систему уравнений при фиксированном $S$, в которой переменные $x_{\vec{n}}^{(S)}$ занумерованы всеми векторами $\vec{n} \in \mathbf{Z}^{r}$. Решение системы (1.5) обозначается через $\left(x_{\vec{n}}^{(S)}(t)\right)$, а в разделе 3 предполагается, что выполнены неравенства (1.6) с константами $\gamma_{S}^{\prime}$ и $\gamma_{S}^{\prime \prime}$.

Результаты статьи были первоначально опубликованы в работах [5].

\section{2. ПРОСТРАНСТВЕННО-ВРЕМЕННОЙ ХАОС И ТЕОРИЯ БЕСКОНЕЧНОМЕРНЫХ СИСТЕМ ОБЫКНОВЕННЫХ ДИФФЕРЕНЦИАЛЬНЫХ УРАВНЕНИЙ}

2.1. Стационарные решения. Решение $\left(\vec{x}_{\vec{n}}^{(S)}\right)=\left(\vec{x}_{\vec{n}}^{(S)}(t)\right)$ системы $(1.5)$ называется стационарным, если оно не зависит от $t$. Пусть $\kappa$ - такое целое число, что $1 \leqslant \kappa \leqslant r$, а $\nu$ - произвольное целое число. Обозначим через $\mathbf{Z}_{\nu}^{(\kappa)}$ множество, состояшее из всех векторов $\vec{n}=\left(n_{1}, \ldots, n_{r}\right) \in \mathbf{Z}^{r}$ таких, что координата $n_{\kappa}=\nu$. Для тех же $\kappa$ и $\nu$ и любого вектора $\vec{n}=\left(n_{1}, \ldots, n_{r}\right) \in \mathbf{Z}^{r}$ введем вектор $\vec{n}^{(\kappa, \nu)}=\left(n_{1}^{(\kappa, \nu)}, \ldots, n_{r}^{(\kappa, \nu)}\right) \in \mathbf{Z}_{\nu}^{(\kappa)}$ такой, что координата $n_{\kappa}^{(\kappa, \nu)}=\nu$, а если $k \neq \kappa$, то $n_{k}^{(\kappa, \nu)}=n_{k}$.

Лемма 2.1. Пусть $\kappa$ - челое число такое, что $1 \leqslant \kappa \leqslant r$, а $\nu$ - фиксированное челое число. Предположим, что для всех векторов $\vec{n}=\left(n_{1}, \ldots, n_{r}\right) \in \mathbf{Z}_{\nu}^{(\kappa)} \cup \mathbf{Z}_{\nu-1}^{(\kappa)}$, заданы числа $\hat{x}_{\vec{n}}$. Тогда справедливы следующие утверждения:

1) существует единственное стационарное решение $\left(\vec{x}_{\vec{n}}^{(S)}\right)$ системь (1.5) такое, что если вектор $\vec{n} \in \mathbf{Z}_{\nu}^{(\kappa)} \cup \mathbf{Z}_{\nu-1}^{(\kappa)}$, то $\vec{x}_{\vec{n}}^{(S)}=\hat{x}_{\vec{n}}$;

2) если для всех $\vec{n} \in \mathbf{Z}_{\nu}^{(\kappa)}$ числа $\hat{x}_{\vec{n}}$ совпадают между собой и для всех $\vec{n} \in$ $\mathbf{Z}_{\nu-1}^{(\kappa)}$ числа $\hat{x}_{\vec{n}}$ совпадают между собой, то при любом значении челого числа $m$ в стационарном решении $\left(\vec{x}_{\vec{n}}^{(S)}\right)$, построенном в $n .1$ данной леммы, для всех $\vec{n} \in \mathbf{Z}_{m}^{(\kappa)}$ числа $\vec{x}_{\vec{n}}^{(S)}$ совпадают между собой.

ДокАЗАТЕльСтво. Стационарное решение $\left(\vec{x}_{\vec{n}}^{(S)}\right)$ системы (1.5) удовлетворяет равенству

$$
\sum_{\vec{n}^{\prime} \in \Gamma_{\vec{n}}} x_{\vec{n}^{\prime}}^{(S)}=2 r x_{\vec{n}}^{(S)}+\frac{1}{2 \chi}\left(f^{(S)}\left(x_{\vec{n}}^{(S)}\right)-\beta^{(S)}\right) .
$$

Поэтому в силу определений вектора $\vec{n}(\kappa, \nu)$, множества $\Gamma_{\vec{n}}$ и $(2.1)$ имеем:

$$
\begin{aligned}
& x_{\vec{n}^{(\kappa, \nu+1)}}^{(S)}=-\sum_{\vec{n}^{\prime} \in \Gamma_{\vec{n}^{(\kappa, \nu)}}^{\prime}} x_{\vec{n}^{\prime}}^{(S)}+2 r x_{\vec{n}^{(\kappa, \nu)}}^{(S)}+\frac{1}{2 \chi}\left(f^{(S)}\left(x_{\vec{n}^{(\kappa, \nu)}}^{(S)}\right)-\beta^{(S)}\right), \\
& x_{\vec{n}^{(\kappa, \nu-2)}}^{(S)}=-\sum_{\vec{n}^{\prime} \in \Gamma_{\vec{n}^{(\kappa, \nu-1)}}^{\prime \prime}} x_{\vec{n}^{\prime}}^{(S)}+2 r x_{\vec{n}^{(\kappa, \nu-1)}}^{(S)}+\frac{1}{2 \chi}\left(f^{(S)}\left(x_{\vec{n}^{(\kappa, \nu-1)}}^{(S)}\right)-\beta^{(S)}\right),
\end{aligned}
$$


где $\Gamma_{\vec{n}^{(\kappa, \nu)}}^{\prime}-$ множество, состояшее из всех векторов $\vec{n}^{\prime} \in \Gamma_{\vec{n}^{(\kappa, \nu)}}$, кроме $\vec{n}^{(\kappa, \nu+1)}$, а $\Gamma_{\vec{n}^{(\kappa, \nu-1)}}^{\prime \prime}-$ множество всех векторов $\vec{n}^{\prime} \in \Gamma_{\vec{n}^{(\kappa, \nu-1)}}$, кроме $\vec{n}^{(\kappa, \nu-2)}$. Заметим, что справедливо включение $\left(\Gamma_{\vec{n}(\kappa, \nu)}^{\prime} \cup \Gamma_{\vec{n}(\kappa, \nu-1)}^{\prime \prime}\right) \subset \mathbf{Z}_{\nu}^{(\kappa)} \cup \mathbf{Z}_{\nu-1}^{(\kappa)}$. Поэтому из равенств (2.2) и (2.3) следует, что если при $\vec{n} \in \mathbf{Z}_{\nu}^{(\kappa)} \cup \mathbf{Z}_{\nu-1}^{(\kappa)}$ компоненты $x_{\vec{n}}^{(S)}$ стационарного решения принимают значения $\vec{x}_{\vec{n}}^{(S)}=\hat{x}_{\vec{n}}$, то через числа $\hat{x}_{\vec{n}}$ однозначно определяются все компоненты

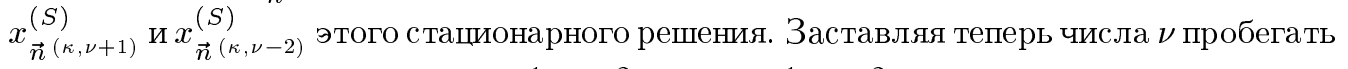
последовательно все значения $\nu+1, \nu+2, \ldots$ и $\nu-1, \nu-2, \ldots$, мы выразим через числа $\hat{x}_{\vec{n}}$ компоненты $x_{\vec{n}}^{(S)}$ этого стационарного решения для всех $\vec{n} \in \mathbf{Z}^{r}$, что и доказывает первое утверждение леммы 2.1. Если теперь для всех $\vec{n} \in \mathbf{Z}_{\nu}^{(\kappa)}$ число $\hat{x}_{\vec{n}}=x_{\vec{n}^{(\kappa, \nu)}}^{(S)}=v$ и для всех $\vec{n} \in \mathbf{Z}_{\nu-1}^{(\kappa)}$ число $\hat{x}_{\vec{n}}=x_{\vec{n}^{(\kappa, \nu-1)}}^{(S)}=u$, то в силу $(2.2)$ при всех $\vec{n} \in \mathbf{Z}_{\nu+1}^{(\kappa)}$

$$
x_{\vec{n}}^{(S)}=-u+2 v+\frac{1}{2 \chi}\left(f^{(S)}(v)-\beta^{(S)}\right),
$$

а в силу (2.3) при всех $\vec{n} \in \mathbf{Z}_{\nu-2}^{(\kappa)}$

$$
x_{\vec{n}}^{(S)}=-v+2 u+\frac{1}{2 \chi}\left(f^{(S)}(u)-\beta^{(S)}\right) .
$$

Таким образом, второе утверждение леммы 2.1 доказано для $m=\nu+1$ и $m=\nu-2$. Заставляя число $\nu$ пробегать последовательно все значения $\nu+1, \nu+2, \ldots$ и $\nu-1$, $\nu-2, \ldots$, мы докажем его для любого целого числа $m$. Лемма 2.1 доказана.

Введем пространство $X$ и его подпространство $X^{0}$ следующим образом: $X \stackrel{\text { def }}{=}\left\{\left(x_{\vec{n}}\right)\right.$ : $\left.\vec{n} \in \mathbf{Z}_{1}^{(1)} \cup \mathbf{Z}_{0}^{(1)}\right\}$, а его точка есть множество $\left(x_{\vec{n}}\right)$ вешественных чисел $x_{\vec{n}}$, занумерованных всеми векторами $\vec{n}$, принадлежашими множеству $\mathbf{Z}_{1}^{(1)} \cup \mathbf{Z}_{0}^{(1)}$. Подпространство $X^{0} \subset X$ состоит из таких множеств $\left(x_{\vec{n}}\right) \in X$, у которых при всех $\vec{n} \in \mathbf{Z}_{1}^{(1)}$ все числа $x_{\vec{n}}$ совпадают между собой, и при всех $\vec{n} \in \mathbf{Z}_{0}^{(1)}$ все числа $x_{\vec{n}}$ совпадают между собой.

Пусть отображение $A:(u, v) \rightarrow\left(u^{\prime}, v^{\prime}\right)$ плоскости $u, v$ таково, что

$$
u^{\prime}=v, \quad v^{\prime}=-u+2 v+\frac{1}{2 \chi}\left(f^{(S)}(v)-\beta^{(S)}\right) .
$$

Лемма 2.2. Рассмотрим точку $\left(x_{\vec{n}}\right) \in X^{0}$ такую, что если $\vec{n} \in \mathbf{Z}_{0}^{(1)}$, то $x_{\vec{n}}=$ $u_{0}$, а если $\vec{n} \in \mathbf{Z}_{1}^{(1)}$, то $x_{\vec{n}}=v_{0}$. Пусть $\left(u_{m}, v_{m}\right)=A^{m}\left(u_{0}, v_{0}\right)$, где $m-$ иелое число. Тогда существует единственное стационарное решение $\left(x_{\vec{n}}^{(S)}\right)$ системь (1.5) такое, что для любого челого числа т при $\vec{n} \in \mathbf{Z}_{m}^{(1)}$ вьполнено равенство $x_{\vec{n}}^{(S)}=u_{m}$, a при $\vec{n} \in \mathbf{Z}_{m+1}^{(1)}$ выполнено равенство $x_{\vec{n}}^{(S)}=v_{m}$.

ДоКАЗАТЕЛЬСтво. Применим лемму 2.1 в частном случае $\kappa=1, \nu=1$. В силу определений $X$ и $X^{0}$, условия леммы 2.2 и леммы 2.1 , сушествует единственное стационарное решение $\left(x_{\vec{n}}^{(S)}\right)$ системы (1.5) такое, что при любом целом $m$ для всех $\vec{n} \in \mathbf{Z}_{m}^{(1)}$ 
числа $x_{\vec{n}}^{(S)}$ совпадают между собой. Более того, из определений вектора $\vec{n}(\kappa, \nu)$ и отображения $A$ и равенств $(2.4),(2.5)$ следует, что для любого $\vec{n} \in \mathbf{Z}^{r}$ и любого целого числа $\nu$ выполняются соотношения

$$
\begin{aligned}
A\left(x_{\vec{n}^{(1, \nu-1)}}^{(S)}, x_{\vec{n}^{(1, \nu)}}^{(S)}\right) & =\left(x_{\vec{n}^{(1, \nu)}}^{(S)}, x_{\vec{n}^{(1, \nu+1)}}^{(S)}\right), \\
A^{-1}\left(x_{\vec{n}^{(1, \nu-1)}}^{(S)}, x_{\vec{n}^{(1, \nu)}}^{(S)}\right) & =\left(x_{\vec{n}^{(1, \nu-2)}}^{(S)}, x_{\vec{n}^{(1, \nu-1)}}^{(S)}\right),
\end{aligned}
$$

в силу равенств $x_{\vec{n}(1,0)}^{(S)}=u_{0}, x_{\vec{n}(1,1)}^{(S)}=v_{0},\left(u_{m}, v_{m}\right)=A^{m}\left(u_{0}, v_{0}\right)$ полностью доказываюшие лемму 2.2 .

Рассмотрим отображение $B:\left(u_{0}, v_{0}\right) \rightarrow\left(x_{\vec{n}}^{(S)}\right)$, которое ставит в соответствие каждой точке $\left(u_{0}, v_{0}\right)$ двумерной плоскости единственное стационарное решение $\left(x_{\vec{n}}^{(S)}\right)$ системы (1.5), построенное в лемме 2.2.

ЛЕмма 2.3. Пусть $\alpha-$ такое число, что

$$
f^{(S)}(\alpha)=\beta^{(S)}, \quad \frac{1}{\chi} \frac{d f^{(S)}}{d x}(\alpha)>0 .
$$

Тогда точка $(\alpha, \alpha)$ - неподвижная точка отображения $A$, имеющая гиперболический mun.

ДоказАТЕльство. В силу определения отображения $A$ при выполнении равенства в системе (2.6) $A(\alpha, \alpha)=(\alpha, \alpha)$, а при выполнении неравенства в этой системе след матрицы линейной части отображения $A$ в точке $(\alpha, \alpha)$ больше, чем 2 , и поскольку определитель этой матрицы равен 1 , то точка $(\alpha, \alpha)$ имеет гиперболический тип. Лемма 2.3 доказана.

Так как неподвижная точка $(\alpha, \alpha)$ отображения $A$ гиперболическая, то у нее есть две сепаратрисы: сжимаюшаяся $\Gamma_{-}$и расширяюшаяся $\Gamma_{+}$. Пусть $\Gamma=\Gamma_{-} \cap \Gamma_{+}-$их пересечение.

Из работы [6] следует, что если входяшая в определение отображения $A$ функция $f^{(S)}(v)$ близка к $\sin v$, а константы $\left|\chi^{-1}\right|$ и $\left|\beta^{(S)} \chi^{-1}\right|$ достаточно малы, то множество Г состоит из бесконечного числа точек.

Введем следующие множества стационарных решений системы (1.5): $\Pi_{-}=B\left(\Gamma_{-}\right)$, $\Pi_{+}=B\left(\Gamma_{+}\right), \Pi=B(\Gamma)$. Тогда имеет место равенство $\Pi=\Pi_{-} \cap \Pi_{+}$.

Из бесконечности множества Г следует, что если функция $f^{(S)}(x)$ близка к $\sin x$, а константы $\left|\chi^{-1}\right|$ и $\left|\beta^{(S)} \chi^{-1}\right|$ достаточно малы, то множество стационарных решений П бесконечно.

2.2. Фазовые пространства. В этом пункте мы введем два типа фазовых пространств системы (1.5), которые потребуются в дальнейшем.

Пусть $\kappa$-целое число, $1 \leqslant \kappa \leqslant r$. Для любых целых чисел $\nu$ и $\mu$ введем пространство

$$
\mathbf{\Omega}_{\nu, \mu}^{(\kappa)}=\left\{\left(g_{\vec{n}}(t)\right): \vec{n} \in \mathbf{Z}_{\nu}^{(\kappa)} \cup \mathbf{Z}_{\mu}^{(\kappa)}\right\},
$$

точкой которого является множество $\left(g_{\vec{n}}(t)\right)$ бесконечно дифференцируемых функций $g_{\vec{n}}(t)$ на прямой $t \in \mathbf{R}^{1}$, занумерованных всеми векторами $\vec{n}$, принадлежащими множеству $\mathbf{Z}_{\nu}^{(\kappa)} \cup \mathbf{Z}_{\mu}^{(\kappa)}$. 
ЛЕмма 2.4. Для любого челого числа $\nu$ пространство $\mathbf{\Omega}_{\nu-1, \nu}^{(\kappa)}$ является фазовым пространством системы (1.5) в следующем смисле: для любой точки $\left(g_{\vec{n}}(t)\right) \in \boldsymbol{\Omega}_{\nu-1, \nu}^{(\kappa)}$ существует единственное решение $\left(x_{\vec{n}}^{(S)}\right)$ системь (1.5) такое, что если $\vec{n} \in \mathbf{Z}_{\nu-1}^{(\kappa)} \cup \mathbf{Z}_{\nu}^{(\kappa)}$, mo $x_{\vec{n}}^{(S)}(t) \equiv g_{\vec{n}}(t)$.

ДокАЗАТЕЛЬСтво. Из равенства (1.5) следует, что

$$
\sum_{\vec{n}^{\prime} \in \Gamma_{\vec{n}}} x_{\vec{n}}^{(S)}(t)=2 r x_{\vec{n}}^{(S)}(t)+\frac{1}{2 \chi}\left(f^{(S)}\left(x_{\vec{n}}^{(S)}(t)\right)-\beta^{(S)}\right)+\frac{d \vec{x}_{\vec{n}}^{(S)}}{d t}(t) .
$$

Отсюда в силу определений вектора $\vec{n}^{(\kappa, \nu)}$ и множества $\Gamma_{\vec{n}}$ получаем

$$
\begin{aligned}
x_{\vec{n}^{(\kappa, \nu+1)}}^{(S)}(t)= & -\sum_{\vec{n}^{\prime} \in \Gamma_{\vec{n}^{(\kappa, \nu)}}^{\prime}} x_{\vec{n}^{\prime}}^{(S)}(t)+2 r x_{\vec{n}^{(\kappa, \nu)}}^{(S)}(t)+ \\
& +\frac{1}{2 \chi}\left(f^{(S)}\left(x_{\vec{n}^{(\kappa, \nu)}}^{(S)}(t)\right)-\beta^{(S)}\right)+\frac{d \vec{x}_{\vec{n}^{(\kappa, \nu)}}^{(S)}(t),}{d t} x_{\vec{n}^{\prime \prime}}^{(S)}(t)+2 r x_{\vec{n}^{(\kappa, \nu-1)}}^{(S)}(t)+ \\
x_{\vec{n}^{(\kappa, \nu-2)}}^{(S)}(t)= & -\sum_{\vec{n}^{\prime \prime} \in \Gamma_{\left.\vec{n}^{\prime \prime}(\kappa)-1\right)}^{\prime \prime}} \\
& +\frac{1}{2 \chi}\left(f^{(S)}\left(x_{\vec{n}^{(\kappa, \nu-1)}}^{(S)}(t)\right)-\beta^{(S)}\right)+\frac{d \vec{x}_{\vec{n}^{(\kappa, \nu-1)}}^{(S)}}{d t}(t),
\end{aligned}
$$

где $\Gamma_{\vec{n}(\kappa, \nu)}^{\prime}$ множество, состояшее из всех векторов множества $\Gamma_{\vec{n}(\kappa, \nu)}$, кроме $\vec{n}^{(\kappa, \nu+1)}$, а $\Gamma_{\vec{n}}^{\prime \prime}(\kappa, \nu-1)-$ множество всех векторов $\Gamma_{\vec{n}(\kappa, \nu-1)}$, кроме $\vec{n}^{(\kappa, \nu-2)}$. Справедливо соотношение

$$
\left(\Gamma_{\vec{n}^{(\kappa, \nu)}}^{\prime} \cup \Gamma_{\vec{n}(\kappa, \nu-1)}^{\prime \prime}\right) \subset\left(\mathbf{Z}_{\nu}^{(\kappa)} \cup \mathbf{Z}_{\nu-1}^{(\kappa)}\right) .
$$

Поэтому из равенств $(2.8)$ и $(2.9)$ следует, что если $\left(x_{\vec{n}}^{(S)}(t)\right)$ - такое решение системы (1.5), что при $\vec{n} \in \mathbf{Z}_{\nu-1}^{(\kappa)} \cup \mathbf{Z}_{\nu}^{(\kappa)}$ выполнено тождество $x_{\vec{n}}^{(S)}(t) \equiv g_{\vec{n}}(t)$, то все компоненты $x_{\vec{n}(\kappa, \nu+1)}^{(S)}(t)$ и $x_{\vec{n}(\kappa, \nu-2)}^{(S)}(t)$ этого решения однозначно определяются заданием точки $\left(g_{\vec{n}}(t)\right) \in \boldsymbol{\Omega}_{\nu-1, \nu}^{(\kappa)}$. Заставляя теперь целое число $\nu$ пробегать последовательно все значения $\nu+1, \nu+2, \ldots$ и $\nu-1, \nu-2, \ldots$, мы однозначно определим все компоненты $x_{\vec{n}}^{(S)}(t)$ решения $\left(x_{\vec{n}}^{(S)}(t)\right)$ при выполнении указанного в лемме условия. Лемма 2.4 доказана.

Рассмотрим $2 r$ целых чисел $\nu_{1}, \mu_{1}, \ldots, \nu_{r}, \mu_{r}$ таких, что

$$
\mu_{k}-\nu_{k}>1, \quad k=1, \ldots, r
$$

Введем множество $Q=Q\left(\nu_{1}, \mu_{1} ; \ldots ; \nu_{r}, \mu_{r}\right) \subset \mathbf{Z}^{r}$, состояшее из таких векторов $\vec{n}=$ $\left(n_{1}, \ldots, n_{r}\right) \in \mathbf{Z}^{r}$, у которых координаты $n_{1}, \ldots, n_{r}$ удовлетворяют неравенствам

$$
\nu_{k}<n_{k}<\mu_{k}, \quad k=1, \ldots, r .
$$


Пусть точкой пространства $\widehat{X}=\widehat{X}\left(\nu_{1}, \mu_{1} ; \ldots ; \nu_{r}, \mu_{r}\right)$ является множество $\left(\hat{x}_{\vec{n}}\right)$, состояшее из вешественных чисел $\hat{x}_{\vec{n}}$, занумерованных всеми векторами $\vec{n} \in Q$.

Обозначим через $\widehat{\boldsymbol{\Omega}}=\widehat{\boldsymbol{\Omega}}\left(\nu_{1}, \mu_{1} ; \ldots ; \nu_{r}, \mu_{r}\right)$ пространство, точкой которого является множество $\left(\omega_{\vec{n}}(t)\right)$ бесконечно дифференцируемых функций $\omega_{\vec{n}}(t)$ на прямой $t \in \mathbf{R}^{1}$, занумерованных всеми векторами $\vec{n} \in \widehat{\mathbf{Z}} \subset \mathbf{Z}^{r}$, где $\widehat{\mathbf{Z}}$ - множество, определенное следующим образом:

$$
\begin{aligned}
& \widehat{\mathbf{Z}}=\widehat{\mathbf{Z}}\left(\nu_{1}, \mu_{1} ; \ldots ; \nu_{r}, \mu_{r}\right) \stackrel{\text { def }}{=} \\
& \stackrel{\text { def }}{=}\left\{\begin{array}{l}
\left(\mathbf{Z}_{\nu_{1}}^{(1)} \cup \mathbf{Z}_{\mu_{1}}^{(1)}\right) \cup\left(\bigcup_{k=2}^{r}\left(\left(\mathbf{Z}_{\nu_{k}}^{(k)} \cup \mathbf{Z}_{\mu_{k}}^{(k)}\right) \cap\left(\bigcap_{m=1}^{k-1}\left(\bigcup_{l=\nu_{m}+1}^{\mu_{m}-1} \mathbf{Z}_{l}^{(m)}\right)\right)\right)\right), \\
\text { если } r \geqslant 2 ; \\
\mathbf{Z}_{\nu_{1}}^{(1)} \cup \mathbf{Z}_{\mu_{1}}^{(1)}, \quad \text { если } r=1 .
\end{array}\right.
\end{aligned}
$$

Наконец, введем пространство $W \stackrel{\text { def }}{=} \widehat{X} \times \widehat{\Omega}$, которое есть прямое произведение пространств $\widehat{X}$ и $\widehat{\Omega}$.

Тогда для множества $\widehat{Q}=Q^{*} \backslash Q$, где $Q^{*}=\left\{\vec{n}=\left(n_{1}, \ldots, n_{r}\right) \in \mathbf{Z}^{r}: \nu_{k} \leqslant n_{k} \leqslant \mu_{k}\right.$; $k=1, \ldots, r\}$, в силу $(2.12)$ имеет место включение $\widehat{Q} \subset \widehat{\mathbf{Z}}$.

ТЕОРема 2.1. Если иелье числа $\nu_{1}, \mu_{1}, \ldots, \nu_{r}, \mu_{r}$ удовлетворяют условию (2.10), то пространство $W=W\left(\nu_{1}, \mu_{1} ; \ldots ; \nu_{r}, \mu_{r}\right)$ является фазовым пространством системы (1.5) в следующем смысле: для любой точки $\left(\hat{x}_{\vec{n}}\right) \in \widehat{X}$, любой точки $\left(\omega_{\vec{n}}(t)\right) \in \widehat{\boldsymbol{\Omega}}$ и любого вещественного числа $t_{0}$ существует единственное решение $\left(x_{\vec{n}}^{(S)}(t)\right)$ системы $(1.5)$ такое, что:

1) если вектор $\vec{n}=\left(n_{1}, \ldots, n_{r}\right) \in Q$, mо $x_{\vec{n}}^{(S)}\left(t_{0}\right)=\hat{x}_{\vec{n}}$;

2) если вектор $\vec{n} \in \widehat{\mathbf{Z}}$, то при всех $t \in \mathbf{R}^{1}$ справедливо равенство $x_{\vec{n}}^{(S)}(t)=\omega_{\vec{n}}(t)$.

ДокАЗАТЕЛЬСТВо. При $\vec{n} \in \widehat{Q}$ положим $x_{\vec{n}}^{(S)}(t)=\omega_{\vec{n}}(t)$ и рассмотрим конечномерную систему

$$
\frac{d \vec{x}_{\vec{n}}^{(S)}}{d t}(t)=-f^{(S)}\left(x_{\vec{n}}^{(S)}\right)+\beta^{(S)}+2 \chi\left(-2 r x_{\vec{n}}^{(S)}+\sum_{\vec{n}^{\prime} \in \Gamma_{\vec{n}}} x_{\vec{n}^{\prime}}^{(S)}\right), \quad n \in Q
$$

состоящую только из тех уравнений системы (1.5), у которых в левой части стоят переменные $x_{\vec{n}}^{(S)}$ с индексами $\vec{n} \in Q$. Согласно классической теореме теории дифференциальных уравнений, сушествует единственное решение $\left(x_{\vec{n}}^{(S)}(t)\right)$ системы $(2.13)$, имеющее компоненты $x_{\vec{n}}^{(S)}(t)=\tilde{x}_{\vec{n}}(t)$, которое удовлетворяет начальным данным $\tilde{x}_{\vec{n}}\left(t_{0}\right)=\hat{x}_{n}$ $(\vec{n} \in Q)$. Докажем, что сушествует единственное решение $\left(x_{\vec{n}}^{(S)}(t)\right)$ системы $(1.5)$ такое, что если $\vec{n} \in Q$, то $x_{\vec{n}}^{(S)}(t) \equiv \tilde{x}_{\vec{n}}(t)$, а если $\vec{n} \in \widehat{\mathbf{Z}}$, то $x_{\vec{n}}^{(S)}(t) \equiv \omega_{\vec{n}}(t)$. С этой целью проведем конечный процесс, состояший из $(r-1)$ последовательных шагов, такой, что если $1 \leqslant p \leqslant r-1$ ( $p$ - целое число), то на $p$-м шаге мы построим единственное решение $\left(x_{\vec{n}}^{(S)}(t)\right)$ системы, состоящей из тех уравнений системы $(1.5)$, у которых в левой части стоят переменные $x_{\vec{n}}^{(S)}$ с индексами $\vec{n} \in \bigcap_{m=1}^{r-p}\left(\bigcup_{l=\nu_{m}+1}^{\mu_{m}-1} \mathbf{Z}_{l}^{(m)}\right)$, и это решение будет удовлетворять следующим условиям: 
а) если $\vec{n} \in Q$, то $x_{\vec{n}}^{(S)}(t) \equiv \tilde{x}_{\vec{n}}(t)$;

б) если $\vec{n} \in \widehat{\mathbf{Z}}$, то $x_{\vec{n}}^{(S)}(t) \equiv \omega_{\vec{n}}(t)$.

Сделаем первьй шаг. Рассмотрим вектор $\vec{n} \in Q$; положим $\kappa=r, \nu=\mu_{r}$ и подставим эти значения в уравнение (2.8). Из определений вектора $\vec{n}^{(\kappa, \nu)}$ и множества $\Gamma_{\vec{n}}^{\prime}(\kappa, \nu)$, входяших в это уравнение, следует, что $\vec{n}^{\left(r, \mu_{r}\right)} \in \widehat{\mathbf{Z}}, \Gamma_{\vec{n}\left(r, \mu_{r}\right)}^{\prime} \subset Q \cup \widehat{Z}$. Поэтому, полагая в правой части уравнения $(2.8) x_{\vec{n}^{(\kappa, \nu)}}^{(S)}(t)=\omega_{\vec{n}\left(r, \mu_{r}\right)}(t)$ и

$$
x_{\vec{n}^{\prime}}^{(S)}(t)=\left\{\begin{array}{lll}
\tilde{x}_{\vec{n}^{\prime}}(t), & \text { если } & \vec{n}^{\prime} \in Q, \\
\omega_{\vec{n}^{\prime}}(t), & \text { если } \vec{n}^{\prime} \in \widehat{\mathbf{Z}},
\end{array}\right.
$$

мы найдем функцию $x_{\vec{n}(\kappa, \nu+1)}^{(S)}(t)=x_{\vec{n}\left(r, \mu_{r}+1\right)}^{(S)}(t)$. Далее, полагая при $\kappa=r$ последовательно $\nu=\mu_{r}+1, \mu_{r}+2, \ldots$, с помошью уравнения (2.8) однозначно определим функции $x_{\vec{n}(\kappa, \nu)}^{(S)}(t)$ для всех $\nu \geqslant \mu_{r}$ через заданные функции $\omega_{\vec{n}}(t)$ и $\tilde{x}_{\vec{n}}(t)$. Точно так же, полагая при $\kappa=r$ последовательно $\nu=\nu_{r}, \nu_{r}-1, \nu_{r}-2, \ldots$, с помощью уравнения (2.9) однозначно определим функции $\left.x_{\vec{n}(\kappa, \nu)}^{(S)}(t)\right)$ при всех $\nu \leqslant \nu_{r}$ через $\omega_{\vec{n}}(t)$ и $\tilde{x}_{\vec{n}}(t)$. Это завершает первый шаг нашего процесса, в результате проведения которого однозначно определяются все функции $x_{\vec{n}}^{(S)}(t)$ при $\vec{n} \in \bigcap_{m=1}^{r-1}\left(\bigcup_{l=\nu_{m}+1}^{\mu_{m}-1} \mathbf{Z}_{l}^{(m)}\right)$ через $\omega_{\vec{n}}(t)$ и $\tilde{x}_{\vec{n}}(t)$. Точно таким же образом, используя уравнения (2.8) и (2.9), в которых мы считаем $\vec{n}$ произвольным вектором $\vec{n} \in \bigcap_{m=1}^{r-p}\left(\bigcup_{l=\nu_{m}+1}^{\mu_{m}-1} \mathbf{Z}_{l}^{(m)}\right), \kappa=r-p+1$, а в качестве $\nu$ берем последовательно значения $\mu_{\kappa}, \mu_{\kappa}+1, \mu_{\kappa}+2, \ldots$ и $\nu_{\kappa}, \nu_{\kappa}-1, \nu_{\kappa}-2, \ldots$, проводится шаг с номером $p$ после шага с номером $p-1$, если $2 \leqslant p \leqslant r-1$. В результате проведения указанных $r-1$ шагов мы однозначно определим функции $x_{\vec{n}}^{(S)}(t)$ при всех $\vec{n} \in \bigcup_{l=\nu_{1}}^{\mu_{1}} \mathbf{Z}_{l}^{(1)}$ через $\tilde{x}_{\vec{n}}(t)$ и $\omega_{\vec{n}}(t)$. Тогда, полагая $\kappa=1, \nu=\nu_{1}+1$ и применяя лемму 2.4 , мы получим утверждение теоремы 2.1 .

2.3. Окрестности стационарных решений и критерий асимптотической устойчивости. Обозначим через $Y$ пространство, произвольной точкой которого является множество $\left(y_{\vec{n}}\right) \in Y$, состояшее из чисел $y_{\vec{n}}$, занумерованных всеми векторами $\vec{n} \in \mathbf{Z}^{r}$. Пусть $x^{0}=\left(x_{\vec{n}}^{0}\right), \vec{n} \in \mathbf{Z}^{r},-$ стационарное решение системы $(1.5)$.

Для произвольного числа $\varepsilon>0$ и любых $2 r$ целых чисел $\nu_{1}, \mu_{1}, \ldots, \nu_{r}, \mu_{r}$, удовлетворяющих неравенствам (2.10), введем окрестность $Y_{\varepsilon}\left(x^{0}\right)=Y_{\varepsilon}\left(x^{0} ; \nu_{1}, \mu_{1} ; \ldots ; \nu_{r}, \mu_{r}\right) \in$ $Y$ стационарного решения $x^{0}$ следуюшим образом: точка $\left(y_{\vec{n}}\right) \in Y_{\varepsilon}\left(x^{0}\right)$ тогда и только тогда, когда

а) $\left|y_{\vec{n}}-x_{\vec{n}}^{0}\right|<\varepsilon$, если $\vec{n} \in Q$,

б) $y_{\vec{n}}=x_{\vec{n}}^{0}$, если $\vec{n} \in \widehat{\mathbf{Z}}$.

Согласно теореме 2.1 , для любой точки $\left(y_{\vec{n}}\right) \in Y_{\varepsilon}\left(x^{0}\right)$ и любого числа $t_{0}$ существует единственное решение $\left(x_{\vec{n}}^{(S)}(t)\right)$ системы $(1.5)$ такое, что если $\vec{n} \in Q$, то $x_{\vec{n}}^{(S)}\left(t_{0}\right)=y_{\vec{n}}$, а если $\vec{n} \in \widehat{\mathbf{Z}}$, то при всех $t \in \mathbf{R}^{1}$ справедливо равенство $x_{\vec{n}}^{(S)}(t)=x_{\vec{n}}^{0}$. Это обстоятельство позволяет говорить, что решение $\left(x_{\vec{n}}^{(S)}(t)\right)$ системы $(1.5)$ при $t=t_{0}$ порождается элементом $\left(y_{\vec{n}}\right) \in Y_{\varepsilon}\left(x^{0}\right)$, если $x_{\vec{n}}^{(S)}\left(t_{0}\right)=y_{\vec{n}}$ при $\vec{n} \in Q$, а при $\vec{n} \in \widehat{\mathbf{Z}}$ для всех $t \in \mathbf{R}^{1}$ справедливо равенство $x_{\vec{n}}^{(S)}(t)=y_{\vec{n}}=x_{\vec{n}}^{0}$. 
Стационарное решение $x^{0}=\left(x_{\vec{n}}^{0}\right)$ системы (1.5) называется асимптотически устойчивым в окрестности $Y_{\varepsilon}\left(x^{0}\right)$, если для любой точки $\left(y_{\vec{n}}\right) \in Y_{\varepsilon}\left(x^{0}\right)$ и любого числа $t_{0}$ решение $\left(x_{\vec{n}}^{(S)}(t)\right)$ системы $(1.5)$, которое при $t=t_{0}$ порождается элементом $\left(y_{\vec{n}}\right)$, при всех $\vec{n} \in \mathbf{Z}^{r}$ удовлетворяет равенству $\lim _{t \rightarrow+\infty} x_{\vec{n}}^{(S)}(t)=x_{\vec{n}}^{0}$.

Следуюшая теорема дает критерий асимптотической устойчивости стационарного решения $x^{0}=\left(x_{\vec{n}}^{0}\right)$ системы $(1.5)$ в окрестности $Y_{\varepsilon}\left(x^{0}\right)$ при достаточно малом $\varepsilon>0$.

Теорема 2.2. Предположим, что параметр х и функиия $f^{(S)}(x)$, входящие в правую часть уравнения (1.5), удовлетворяют следующим неравенствам:

$$
\chi>0, \quad a_{\vec{n}} \stackrel{\text { def }}{=} \frac{d f^{(S)}}{d x}\left(x_{\vec{n}}^{0}\right)>0, \quad \vec{n} \in Q .
$$

Тогда существует такое $\varepsilon_{0}>0$, что если $0<\varepsilon \leqslant \varepsilon_{0}$, то стационарное решение $x^{0}=\left(x_{\vec{n}}^{0}\right)$ асимптотически устойчиво в окрестности $Y_{\varepsilon}\left(x^{0}\right)$.

Докажем сначала вспомогательную лемму.

Лемма 2.5. Рассмотрим решение $\left(x_{\vec{n}}^{(S)}(t)\right), \quad \vec{n} \in Q$, с начальныцми данными $x_{\vec{n}}^{(S)}\left(t_{0}\right)$ при $t=t_{0}$ конечномерной системы $(2.13)$, в которой мы полагаем $x_{\vec{n}}^{(S)}(t) \equiv$ $x_{\vec{n}}^{0}$, если $\vec{n} \in \widehat{Q}$. Тогда существует такое $\varepsilon_{0}>0$, что если $0<\varepsilon \leqslant \varepsilon_{0}$ и при $\vec{n} \in Q$ справедливы неравенства $\left|x_{\vec{n}}^{(S)}\left(t_{0}\right)-x_{\vec{n}}^{0}\right|<\varepsilon$, то при всех $\vec{n} \in Q$ выполняются следующие утверждения:

1) $\lim _{t \rightarrow+\infty} x_{\vec{n}}^{(S)}(t)=x_{\vec{n}}^{0}$

2) для любого натурального числа $m \lim _{t \rightarrow+\infty} d^{m} x_{\vec{n}}^{(S)}(t) / d t^{m}=0$.

ДокАЗАТЕЛЬСТво. Для доказательства леммы рассмотрим квадратичную форму

$$
\widetilde{V}=\widetilde{V}\left(\left(y_{n}\right)\right) \stackrel{\text { def }}{=} \sum_{\vec{n} \in Q} a_{\vec{n}} y_{\vec{n}}^{2}+\chi \sum_{\begin{array}{c}
\left(\vec{n}^{\prime}, \vec{n}^{\prime \prime}\right) \in \Gamma \\
\vec{n}^{\prime} \in Q^{*} \\
\vec{n}^{\prime \prime} \in Q^{*}
\end{array}}\left(y_{\vec{n}^{\prime}}-y_{\vec{n}^{\prime \prime}}\right)^{2},
$$

где полагаем $y_{\vec{n}^{\prime}}=0$ или $y_{\vec{n}^{\prime \prime}}=0$, если $\vec{n}^{\prime} \in \widehat{Q}$ или $\vec{n}^{\prime \prime} \in \widehat{Q}$. В силу неравенства $(2.14)$ форма $\widetilde{V}$ положительно определена, и поэтому все собственные числа ее матришы $\widehat{V}$ вешественные и положительные. Но из вида системы (2.13) следует, что матрица $\widehat{\mathcal{U}}$ ее линейной части в неподвижной точке $\left(x_{\vec{n}}^{0}\right)$ имеет вид $\widehat{\mathcal{U}}=-\widehat{V}$; следовательно, все собственные числа матрицы $\widehat{\mathcal{U}}$ - вешественные и отрицательные. Это означает, что точка $\left(x_{\vec{n}}^{0}\right)$ асимптотически устойчива по Ляпунову, что и доказывает первое утверждение леммы 2.5. Далее, из вида системы (2.13) следует, что ее решение бесконечно дифференцируемо. Докажем, что при $m=1$ справедливо второе утверждение леммы. Действительно, в силу (2.13) все первые производные функций $x_{\vec{n}}^{(S)}(t)$ выражаются через сами эти функции. Поэтому, переходя к пределу при $t \rightarrow+\infty$ в правой части равенства $(2.13)$ и учитывая, что $\left(x_{\vec{n}}^{0}\right)$ - стационарное решение системы (1.5), мы докажем утверждение 2 леммы 2.5. Если же $m>1$, то, дифференцируя $(2.13)(m-1)$ раз, получим, что 
все производные $d^{m} x_{\vec{n}}^{(S)}(t) / d t^{m}$ выражаются через производные функций $x_{\vec{n}}^{(S)}(t)$ меньших порядков. Предполагая, что для производных функций $x_{\vec{n}}^{(S)}(t)$, имеющих порядок меньше $m$, справедливо утверждение 2 , мы докажем его для всех натуральных чисел $m$. Лемма 2.5 доказана.

Пусть $\varepsilon_{0}-$ число, для которого справедливы утверждения леммы $2.5,0<\varepsilon \leqslant \varepsilon_{0}$, $\left(y_{\vec{n}}\right) \in Y_{\varepsilon}\left(x^{0}\right)$ и $\left(x_{\vec{n}}^{(S)}(t)\right)$ - решение системы $(1.5)$, порожденное элементом $\left(y_{\vec{n}}\right)$. При $\vec{n} \in Q$ компонента $x_{\vec{n}}^{(S)}(t)$ решения системы (1.5) совпадает с соответствуюшей компонентой $x_{\vec{n}}^{(S)}(t)$ решения $\left(x_{\vec{n}}^{(S)}(t)\right)$ системы $(2.13)$, в которой при $\vec{n} \in \widehat{Q}$ справедливы равенства $x_{\vec{n}}^{(S)}(t)=x_{\vec{n}}^{0}$. Более того, из доказательства теоремы 2.1 следует, что при любом $\vec{n} \in \mathbf{Z}^{r}$ компонента $x_{\vec{n}}^{(S)}(t)$ решения системы (1.5) однозначно выражается через компоненты решения конечномерной системы из леммы 2.5 , их производные и компоненты $x_{\vec{n}}^{0}$ стационарного решения так, что если при $\vec{n} \in Q$ выполнены равенства $\lim _{t \rightarrow+\infty} x_{\vec{n}}^{(S)}(t)=x_{\vec{n}}^{0}$, а для любого натурального числа $m$ выполнены равенства $\lim _{t \rightarrow+\infty} d^{m} x_{\vec{n}}^{(S)}(t) / d t^{m}=0$, то при любом $\vec{n} \in \mathbf{Z}^{r}$ вьполнено равенство $\lim _{t \rightarrow+\infty} x_{\vec{n}}^{(S)}(t)=x_{\vec{n}}^{0}$. Поэтому утверждение теоремы 2.2 следует из леммы 2.5 и определения асимптотически устойчивого стационарного решения системы (1.5). Теорема 2.2 доказана.

2.4. Построение бесконечномерных странных аттракторов. На протяжении этого пункта будем предполагать, что параметры $\chi, \beta^{(S)}$ и функция $f^{(S)}(x)$, входящие в систему уравнений (1.5), удовлетворяют следующим условиям:

а) константа $\chi>0$;

б) существует такое число $\alpha$, что $f^{(S)}(\alpha)=\beta^{(S)}, d f^{(S)}(\alpha) / d x>0$.

Так как при выполнении этих условий справедливы и условия (2.6), то в силу леммы 2.3 сушествуют семейства П, П-, П + стационарных решений системы (1.5). Главная наша цель на этом этапе состоит в том, чтобы применить результат п. 2.3 (теорему 2.2) к этим семействам.

ТЕОРема 2.3. Пусть стационарное решение системы (1.5) $x^{0}=\left(x_{\vec{n}}^{0}\right) \in \Pi$; $\nu_{1}, \mu_{1}, \ldots, \nu_{r}, \mu_{r}-2 r$ чельх чисел таких, что

$$
\mu_{k}=\nu_{k}+N_{k}+1, \quad N_{k} \geqslant 1, \quad k=1, \ldots, r .
$$

Тогда существуют числа $M_{-}$и $M_{+}$имножество положительных чисел $\varepsilon_{\vec{m}}$, занумерованных всеми векторами $\vec{m}=\left(m_{1}, \ldots, m_{r}\right)$ с натуральнылми координатами $m_{k}, k=1, \ldots, r$, такие, что если выполнено хотя бы одно из неравенств

$$
\nu_{1} \geqslant M_{+}, \quad \mu_{1} \leqslant M_{-}
$$

то стационарное решение $x^{0}$ асимптотически устойчиво в окрестности $Y_{\varepsilon_{\vec{m}^{0}}}\left(x^{0} ; \nu_{1}, \mu_{1} ; \ldots ; \nu_{r}, \mu_{r}\right)$, где $\vec{m}^{0}=\left(m_{1}^{0}, \ldots, m_{r}^{0}\right)-$ вектор с координатами $m_{k}^{0}=$ $N_{k} \quad k=1, \ldots, r$. 
ДоказАтельство. Так как стационарное решение $\left(x_{\vec{n}}^{0}\right) \in \Pi$, то в силу лемм $2.2,2.3$ для любого $\delta>0$ сушествуют константы $M_{-}^{(\delta)}$ и $M_{+}^{(\delta)}$ такие, что если целое число $m$ удовлетворяет хотя бы одному из двух неравенств $m \geqslant M_{+}^{(\delta)}, m \leqslant M_{-}^{(\delta)}$, то при $\vec{n} \in \mathbf{Z}_{m}^{(1)}$ выполнено неравенство $\left|x_{\vec{n}}^{0}-\alpha\right|<\delta$. Из условия существования числа $\alpha$, сформулированного в начале этого пункта, следует, что сушествует число $\delta_{0}>0$ такое, что в области

$$
|x-\alpha|<\delta_{0}
$$

выполняется неравенство

$$
\frac{d f^{(S)}}{d x}(x)>0 .
$$

Полагая $M_{-}=M_{-}^{\left(\delta_{0}\right)}, M_{+}=M_{+}^{\left(\delta_{0}\right)}$ и предполагая, что справедливо хотя бы одно из двух неравенств $(2.17)$, в силу неравенств $\chi>0$ и (2.19) и определения множества $Q$ получим, что справедливы неравенства (2.14). Поэтому, согласно теореме 2.2 , сушествует такое $\varepsilon_{0}>0$, что если $0<\varepsilon \leqslant \varepsilon_{0}$, то стационарное решение $x^{0}=\left(x_{\vec{n}}^{0}\right)$ асимптотически устойчиво в окрестности $Y_{\varepsilon}\left(x^{0} ; \nu_{1}, \mu_{1} ; \ldots ; \nu_{r}, \mu_{r}\right)$. Но из доказательства теоремы 2.2 и неравенства (2.18) следует, что число $\varepsilon_{0}>0$ зависит только от чисел $N_{k}, k=1, \ldots, r$, введенных в (2.16), и не зависит от $\nu_{1}, \mu_{1}, \ldots, \nu_{r}, \mu_{r}$, если выполнено хотя бы одно из неравенств (2.17). Таким образом, мы можем считать $\varepsilon_{0}=\varepsilon_{\vec{m}^{0}}$. Теорема 2.3 доказана.

ТеОрема 2.4. Пусть стационарное решение системы $(1.5) x^{0}=\left(x_{\vec{n}}^{0}\right) \in \Pi_{+}($или $\left.x^{0} \in \Pi_{-}\right) ; \quad \nu_{1}, \mu_{1}, \ldots, \nu_{r}, \mu_{r}-2 r$ чельх чисел, удовлетворяющих условию (2.16). Тогда существуют число $M_{-}$(соответственно $\left.M_{+}\right)$и мнохсество полохстельных чисел $\varepsilon_{\vec{m}}$, занумерованных всеми векторами $\vec{m}=\left(m_{1}, \ldots, m_{r}\right)$ с натуральными координатами $m_{k}, k=1, \ldots, r$, такие, что если выполнено неравенство $\mu_{1} \leqslant M_{-}$(соответственно $\left.\nu_{1} \geqslant M_{+}\right)$, то стационарное речение $x^{0}$ асимптотически устойчиво в окрестности $Y_{\varepsilon_{\vec{m}^{0}}}\left(x^{0} ; \nu_{1}, \mu_{1} ; \ldots ; \nu_{r}, \mu_{r}\right)$, где $\vec{m}^{0}=\left(m_{1}^{0}, \ldots, m_{r}^{0}\right)$ вектор с координатами $m_{k}^{0}=N_{k}, a N_{k}, k=1, \ldots, r,-$ числа, введеннье в (2.16). При этом, если $x^{0} \in \Pi$, то числа $M_{-}, M_{+} u \varepsilon_{\vec{m}}$ совпадают с соответствующими числами, найденными в теореме 2.3 .

ДокаЗАтельство. Так как стационарное решение $x^{0} \in \Pi_{+}\left(\right.$или $\left.x^{0} \in \Pi_{-}\right)$, то в силу лемм $2.2,2.3$ для любого $\delta>0$ сушествует константа $M_{-}^{(\delta)}$ (соответственно $M_{+}^{(\delta)}$ ) такая, что если целое число $m$ удовлетворяет неравенству $m<M_{-}^{(\delta)}$ (соответственно $\left.m>M_{+}^{(\delta)}\right)$, то при $\vec{n} \in \mathbf{Z}_{m}^{(1)}$ выполняется неравенство $\left|x_{\vec{n}}^{0}-\alpha\right|<\delta$. Так же, как в теореме 2.2 , выберем в качестве $\delta$ число $\delta=\delta_{0}>0$ такое, что в области $(2.18)$ справедливо неравенство (2.19), положим $M_{-}=M_{-}^{\left(\delta_{0}\right)}$ (соответственно $\left.M_{+}=M_{+}^{\left(\delta_{0}\right)}\right)$ и, считая $\mu_{1} \leqslant M_{-}$(соответственно $\left.\nu_{1} \geqslant M_{+}\right)$, получим, что справедливо условие (2.14) теоремы 2.2. Далее вывод утверждения теоремы 2.4 из теоремы 2.2 полностью повторяет вывод утверждения теоремы 2.3. Теорема 2.4 доказана.

Пусть $x^{0}$ - стационарное решение системы (1.5); $t_{0}$ - произвольное число; $\nu_{1}, \mu_{1}, \ldots, \nu_{r}, \mu_{r}-2 r$ целых чисел, удовлетворяющих условию (2.16). Для любого $\varepsilon>0$ определим множество $V_{\varepsilon}=V_{\varepsilon}\left(x^{0} ; t_{0} ; \nu_{1}, \mu_{1} ; \ldots ; \nu_{r}, \mu_{r}\right)$, состоящее из таких 
решений системы (1.5), которые при $t=t_{0}$ порождаются всеми точками окрестности $Y_{\varepsilon}\left(x^{0} ; \nu_{1}, \mu_{1} ; \ldots ; \nu_{r}, \mu_{r}\right)$.

Введем множества $V_{+}\left(x_{0}\right), V_{-}\left(x_{0}\right), V\left(x_{0}\right)$ решений системы (1.5) следуюшим обра30м:

1) если $x^{0} \in \Pi_{+}$, то

$$
V_{+}\left(x^{0}\right)=\bigcup_{\substack{\mu_{1} \leqslant M_{-} \\\left(\nu_{1}, \mu_{1}, \ldots, \nu_{r}, \mu_{r}\right)}} V_{\varepsilon_{\vec{m}}}\left(x^{0} ; t_{0} ; \nu_{1}, \mu_{1} ; \ldots ; \nu_{r}, \mu_{r}\right) ;
$$

2) если $x^{0} \in \Pi_{-}$, то

$$
V_{-}\left(x^{0}\right)=\bigcup_{\substack{\nu_{1} \geqslant M_{+} \\\left(\nu_{1}, \mu_{1}, \ldots, \nu_{r}, \mu_{r}\right)}} V_{\varepsilon_{\vec{m}}}\left(x^{0} ; t_{0} ; \nu_{1}, \mu_{1} ; \ldots ; \nu_{r}, \mu_{r}\right) ;
$$

3) если $x^{0} \in \Pi$, то $V\left(x^{0}\right)=V_{+}\left(x^{0}\right) \cup V_{-}\left(x^{0}\right)$,

где объединения проводятся по всем наборам $\left(\nu_{1}, \mu_{1}, \ldots, \nu_{r}, \mu_{r}\right)$, состояшим из $2 r$ целых чисел таких, что в случае $1 \mu_{1} \leqslant M_{-}$, в случае $2 \nu_{1} \geqslant M_{+}$и выполнено условие (2.16), а $M_{-}, M_{+}, \varepsilon_{\vec{m}}, \vec{m}$ - числа и векторы, определенные в теоремах 2.3 и 2.4.

Будем считать, что решение $\left(x_{\vec{n}}^{(S)}(t)\right)$ системы $(1.5)$ при $t \rightarrow+\infty$ сходится к ее стационарному решению $\left(x_{\vec{n}}^{0}\right)$, если при всех $\vec{n} \in \mathbf{Z}^{r}$ справедливо равенство $\lim _{t \rightarrow+\infty} x_{\vec{n}}^{(S)}(t)=$ $x_{\vec{n}}^{0}$.

Тогда из теорем 2.3 и 2.4 , а также из того факта, что размерность множества $V_{\varepsilon}$ равна $\prod_{k=1}^{r} N_{k}$, непосредственно вытекает

СЛЕДСТВИЕ 2.1. Множества $V\left(x^{0}\right), V_{+}\left(x^{0}\right)$ u $V_{-}\left(x^{0}\right)$ бесконечномерны и таковы, что если выполняется хотя би одно из трех условий $\left(x_{\vec{n}}^{(S)}(t)\right) \in V\left(x^{0}\right)$, $\left(x_{\vec{n}}^{(S)}(t)\right) \in V_{+}\left(x^{0}\right), \quad\left(x_{\vec{n}}^{(S)}(t)\right) \in V_{-}\left(x^{0}\right)$, то решение $\left(x_{\vec{n}}^{(S)}(t)\right)$ при $t \rightarrow+\infty$ сходится $\kappa$ стачионарному решению $x^{0}$.

\section{3. КРИТИЧЕСКИЕ ЯВЛЕНИЯ И БИФУРКАЦИИ РЕШЕНИЙ БЕСКОНЕЧНОМЕРНЫХ СИСТЕМ ОБЫКНОВЕННЫХ ДИФФЕРЕНЦИАЛЬНЫХ УРАВНЕНИЙ}

3.1. Бифуркации пространственно-однородных решений относительно изменения параметров. Решение $\left(\vec{x}_{\vec{n}}^{(S)}(t)\right)$ системы $(1.5)$ называется пространственно-однородным, если функция $x_{\vec{n}}^{(S)}(t)$ не зависит от вектора $\vec{n} \in \mathbf{Z}$.

Зафиксируем функцию $f^{(S)}(x)$ и параметр $\chi$ в системе $(1.5)$ и будем изучать поведение пространственно-однородных решений в зависимости от параметра $\beta^{(S)}$.

ТЕОРема 3.1. Пусть $\vec{x}^{(S)}=\left(x_{\vec{n}}^{(S)}(t)\right)$ - пространственно-однородное решение. Тогда справедливь следующие утверждения.

1. Eсли $\beta^{(S)}>\gamma_{S}^{\prime \prime}$, mo $\lim _{t \rightarrow+\infty} x_{\vec{n}}^{(S)}(t)=+\infty, d x_{\vec{n}}^{(S)}(t) / d t>0$, аесли $\beta^{(S)}<\gamma_{S}^{\prime}$, $m o \lim _{t \rightarrow+\infty} x_{\vec{n}}^{(S)}(t)=-\infty, d x_{\vec{n}}^{(S)}(t) / d t<0$. 
2. Предположим, что въполнено хотя бъ одно из следующих условий:

а) $\gamma_{S}^{\prime} \leqslant \beta^{(S)} \leqslant \gamma_{S}^{\prime \prime}$ и начальное значение $x_{0}^{(S)}=x_{\vec{n}}^{(S)}\left(t_{0}\right)$ удовлетворяет неравенствам $f^{(S)}\left(x_{0}^{(S)}\right) \leqslant \beta^{(S)}, \quad x_{0}^{(S)} \leqslant \bar{x}^{(S)}$, где $\bar{x}^{(S)}$ - такое число, что $f^{(S)}\left(\bar{x}^{(S)}\right) \geqslant$ $\beta^{(S)}$

б) $\gamma_{S}^{\prime} \leqslant \beta^{(S)} \leqslant \gamma_{S}^{\prime \prime}$ и начальное значение $x_{0}^{(S)}=x_{\vec{n}}^{(S)}\left(t_{0}\right)$ удовлетворяет неравенствам $f^{(S)}\left(x_{0}^{(S)}\right) \geqslant \beta^{(S)}, \quad x_{0}^{(S)} \geqslant \tilde{x}^{(S)}$, где $\tilde{x}^{(S)}-$ такое число, что $f^{(S)}\left(\tilde{x}^{(S)}\right) \leqslant$ $\beta^{(S)}$.

Тогда решение $x_{\vec{n}}^{(S)}(t)$ с начальными данными $x_{0}^{(S)}=x_{\vec{n}}^{(S)}\left(t_{0}\right)$ имеет конечный предел $\hat{x}=\lim _{t \rightarrow+\infty} x_{\vec{n}}^{(S)}(t)$.

Для доказательства теоремы рассмотрим уравнение

$$
\frac{d x}{d t}=-f^{(S)}(x)+\beta^{(S)}
$$

и докажем три леммы о свойствах его решений.

ЛЕмма 3.1. Eсли $\beta^{(S)}>\gamma_{S}^{\prime \prime}$, mo $\lim _{t \rightarrow+\infty} x(t)=+\infty, d x(t) / d t>0$, a если $\beta^{(S)}<$ $\gamma_{S}^{\prime}$, mo $\lim _{t \rightarrow+\infty} x(t)=-\infty, d x(t) / d t<0$.

ДокАЗАТЕЛЬство. Утверждение леммы 3.1 очевидно следует из неравенств (1.6).

ЛЕмма 3.2. Предположим, что $\gamma_{S}^{\prime} \leqslant \beta^{(S)} \leqslant \gamma_{S}^{\prime \prime}$ и начальное значение $x_{0}=x\left(t_{0}\right)$ удовлетворяет неравенствам $f^{(S)}\left(x_{0}\right) \leqslant \beta^{(S)}, x_{0} \leqslant \bar{x}$, дде $\bar{x}-$ такое число, что $f^{(S)}(\bar{x}) \geqslant \beta^{(S)}$. Тогда решение $x(t)$ уравнения $(3.1)$ с начальным значением $x_{0}=$ $x\left(t_{0}\right)$ имеет конечный предел $\hat{x}=\lim _{t \rightarrow+\infty} x(t)<\infty$.

ДокАЗАТЕЛЬСТво. Из неравенств $x_{0} \leqslant \bar{x}, f^{(S)}\left(x_{0}\right) \leqslant \beta^{(S)}, f^{(S)}(\bar{x}) \geqslant \beta^{(S)}$ следует, что существует число $\overline{\bar{x}}$, удовлетворяющее условиям $x_{0} \leqslant \overline{\bar{x}} \leqslant \bar{x}, f^{(S)}(\overline{\bar{x}})=\beta^{(S)}$, такое, что в интервале $\left(x_{0}, \overline{\bar{x}}\right)$ нет чисел $\xi$, для которых $f^{(S)}(\xi)=\beta^{(S)}$. Докажем, что $\lim _{t \rightarrow+\infty} x(t)=\overline{\bar{x}}$. Согласно неравенству $f^{(S)}\left(x_{0}\right) \leqslant \beta^{(S)}$ и выбору числа $\overline{\bar{x}}$, в интервале $x_{0}<x<\overline{\bar{x}}$ выполнено неравенство $\beta^{(S)}-f^{(S)}(x)>0$, и решение $x(t)$ уравнения $(3.1)$ будет возрастать с ростом $t$. Поэтому для любого $\epsilon>0$ существует значение $\overline{\bar{t}} \geqslant t_{0}$ такое, что $\overline{\bar{x}}-\epsilon \leqslant x(\overline{\bar{t}}) \leqslant \overline{\bar{x}}$. Представим функцию $f^{(S)}(x)$ в окрестности точки $x=\overline{\bar{x}}$ в виде

$$
f^{(S)}(x)=\beta^{(S)}+a_{\nu}(x-\overline{\bar{x}})^{\nu}+O\left(|x-\overline{\bar{x}}|^{\nu+1}\right),
$$

где $\nu$ - натуральное число, $a_{\nu}$ - константа и $\left|O\left(|x-\overline{\bar{x}}|^{\nu+1}\right)\right| \leqslant$ const $|x-\overline{\bar{x}}|^{\nu+1}$. Из этого представления следует, что при достаточно малом $\epsilon>0$

$$
\int_{\overline{\bar{x}}-\epsilon}^{\overline{\bar{x}}} \frac{d x}{\beta^{(S)}-f^{(S)}(x)}=\infty .
$$

Поэтому в силу (3.1) либо для любого конечного числа $t \geqslant t_{0}$ выполнено неравенство $x(t)<\overline{\bar{x}}$, либо $x(t) \equiv \overline{\bar{x}}$ при всех $t \geqslant t_{0}$. Лемма 3.2 доказана. 
ЛЕмма 3.3. Предположим, что $\gamma_{S}^{\prime} \leqslant \beta^{(S)} \leqslant \gamma_{S}^{\prime \prime}$ и начальное значение $x_{0}=x\left(t_{0}\right)$ удовлетворяет неравенствам $f^{(S)}\left(x_{0}\right) \geqslant \beta^{(S)}, x_{0} \geqslant \tilde{x}$, где $\tilde{x}-$ такое число, что $f^{(S)}(\tilde{x}) \leqslant \beta^{(S)}$. Тогда решение $x(t)$ уравнения (3.1) с начальным значением $x_{0}$ имеет конечный предел $\hat{x}=\lim _{t \rightarrow+\infty} x(t)$.

Доказательство леммы 3.3 проводится так же, как доказательство леммы 3.2.

Так как $\left(x_{\vec{n}}^{(S)}(t)\right)$ - пространственно-однородное решение системы $(1.5)$, то для всех векторов $\vec{n} \in \mathbf{Z}^{r}$ функция $x_{\vec{n}}^{(S)}(t)$ равна некоторой функции $x(t)$. Подставляя $x_{\vec{n}}^{(S)}(t)=$ $x(t)$ в уравнение (1.5), получим уравнение (3.1) для функции $x(t)$. Теперь утверждение 1 теоремы 3.1 следует из леммы 3.1, а утверждение 2 следует из лемм 3.2 и 3.3. Теорема 3.1 доказана.

СлеДСТВИЕ 3.1. Предположим, что функиия $f^{(S)}(x)$ периодическая $u\left(x_{\vec{n}}^{(S)}(t)\right)$ пространственно-однородное решение системы (1.5). Тогда имеет место один из следующих трех случаев:

1) если $\gamma_{S}^{\prime} \leqslant \beta^{(S)} \leqslant \gamma_{S}^{\prime \prime}$, mо $\lim _{t \rightarrow+\infty} x_{\vec{n}}^{(S)}(t)=\hat{x}$, где $\hat{x}-$ конечное число;

2) если $\beta^{(S)}>\gamma_{S}^{\prime \prime}$, mo $\lim _{t \rightarrow+\infty} x_{\vec{n}}^{(S)}(t)=+\infty$;

3) если $\beta^{(S)}<\gamma_{S}^{\prime}$, mo $\lim _{t \rightarrow+\infty} x_{\vec{n}}^{(S)}(t)=-\infty$.

СЛЕДСТВИЕ 3.2. Если функиия $f^{(S)}(x)$ периодическая, то $\beta^{(S)}=\gamma_{S}^{\prime}$ и $\beta^{(S)}=$ $\gamma_{S}^{\prime \prime}$ - критические значения параметра $\beta^{(S)}$, при прохождении которых поведение пространственно-однородного решения системы (1.5) принципиально меняется $n p u t \rightarrow+\infty$.

3.2. Бифуркации решений относительно изменения начальных данных. В этом пункте мы найдем семейство решений системы (1.5), которые не являются пространственно-однородными, и изучим их бифуркации при изменении начальных данных.

ТЕОрема 3.2. Предположим, что функиия $f^{(S)}(x)$ и константа $\beta^{(S)}$ удовлетворяют неравенству

$$
2 \beta^{(S)} \equiv f^{(S)}(x)+f^{(S)}(-x) .
$$

Тогда для любого числа $x_{0}^{(S)}$ существует решение $\left(x_{\vec{n}}^{(S)}(t)\right)$ системы (1.5) такое, что для любих двух векторов $\vec{n}^{\prime}=\left(n_{1}^{\prime}, \ldots, n_{r}^{\prime}\right) \in \mathbf{Z}^{r} u \vec{n}^{\prime \prime}=\left(n_{1}^{\prime \prime}, \ldots, n_{r}^{\prime \prime}\right) \in \mathbf{Z}^{r}$, чьи координаты удовлетворяют равенству

$$
\sum_{\nu=1}^{r}\left|n_{\nu}^{\prime}-n_{\nu}^{\prime \prime}\right|=1,
$$

верно тохсдество

$$
x_{\vec{n}^{\prime}}^{(S)}(t) \equiv-x_{\vec{n}^{\prime \prime}}^{(S)}(t)
$$

в начальный момент времени $t=t_{0}$ выполнено одно из равенств $x_{\vec{n}}^{(S)}\left(t_{0}\right)=x_{0}^{(S)}$ и $x_{\vec{n}}^{(S)}\left(t_{0}\right)=-x_{0}^{(S)}$ и справедливы следующие утверәсдения:

1) если $\chi \leqslant 0$ u $8 r \chi x_{0}^{(S)}<\beta^{(S)}-\gamma_{S}^{\prime \prime}$, mo $\lim _{t \rightarrow+\infty}\left|x_{\vec{n}}^{(S)}(t)\right|=+\infty$;

2) если $\chi \geqslant 0$ u $8 r \chi x_{0}^{(S)}>\beta^{(S)}-\gamma_{S}^{\prime}$, mo $\lim _{t \rightarrow+\infty}\left|x_{\vec{n}}^{(S)}(t)\right|=+\infty$; 
3) если $\beta^{(S)}-\gamma_{S}^{\prime \prime} \leqslant 8 r \chi x_{0}^{(S)} \leqslant \beta^{(S)}-\gamma_{S}^{\prime}, \beta^{(S)}-f^{(S)}\left(x_{0}^{(S)}\right) \geqslant 8 r \chi x_{0}^{(S)}$ u cyществует такое число $\bar{x}^{(S)} \geqslant x_{0}^{(S)}$, что $\beta^{(S)}-f^{(S)}\left(\bar{x}^{(S)}\right) \leqslant 8 r \chi x_{0}^{(S)}$, mo $\lim _{t \rightarrow+\infty} x_{\vec{n}}^{(S)}(t)=$ $\hat{x}^{(S)}$, где $\hat{x}^{(S)}$ - конечное число;

4) если $\beta^{(S)}-\gamma_{S}^{\prime \prime} \leqslant 8 r \chi x_{0}^{(S)} \leqslant \beta^{(S)}-\gamma_{S}^{\prime}, \beta^{(S)}-f^{(S)}\left(x_{0}^{(S)}\right) \leqslant 8 r \chi x_{0}^{(S)}$ и существует такое число $\tilde{x}^{(S)} \leqslant x_{0}^{(S)}$, что $\beta^{(S)}-f^{(S)}\left(\tilde{x}^{(S)}\right) \geqslant 8 r \chi \tilde{x}^{(S)}$, mo $\lim _{t \rightarrow+\infty} x_{\vec{n}}^{(S)}(t)=$ $\hat{x}^{(S)}$, дде $\hat{x}^{(S)}$ - конечное число.

Для доказательства теоремы рассмотрим уравнение

$$
\frac{d y}{d t}=-f^{(S)}(y)+\beta^{(S)}-8 r \chi y
$$

и докажем четыре леммы о свойствах его решений.

ЛЕмма 3.4. Если $\chi \leqslant 0$ и начальное значение $y_{0}=y\left(t_{0}\right)$ удовлетворяет неравенству

$$
8 r \chi y_{0}<\beta^{(S)}-\gamma_{S}^{\prime \prime}
$$

$m o \lim _{t \rightarrow+\infty} y(t)=+\infty$.

ДоказАТЕЛьСТво. В силу (1.6), (3.7) и неравенства $\chi \leqslant 0$ при всех $y \geqslant y_{0}$ справедливы неравенства $-f^{(S)}(y)+\beta^{(S)}-8 r \chi y \geqslant-\gamma_{S}^{\prime \prime}+\beta^{(S)}-8 r \chi y_{0}>C^{\prime}>0$, где $C^{\prime}-$ константа.

Поэтому согласно (3.6) при всех $t \geqslant t_{0}$ выполнено неравенство $d y(t) / d t \geqslant C^{\prime}>0$, что и доказывает лемму 3.4 .

ЛЕмма 3.5. Если $\chi \geqslant 0$ и начальное значение $y_{0}=y\left(t_{0}\right)$ удовлетворяет неравенству

$$
8 r \chi y_{0}>\beta^{(S)}-\gamma_{S}^{\prime}
$$

$m o \lim _{t \rightarrow+\infty} y(t)=-\infty$.

ДоКАЗАТЕЛЬСТВО проводится так же, как и доказательство леммы 3.4.

Лемма 3.6. Предположим ито ито $\beta^{(S)}-\gamma_{S}^{\prime \prime} \leqslant 8 r \chi y_{0} \leqslant \beta^{(S)}-\gamma_{S}^{\prime}, \beta^{(S)}-f^{(S)}\left(y_{0}\right) \geqslant$ $8 r \chi y_{0}$ и существует число $\bar{y} \geqslant y_{0}$ такое, что $\beta^{(S)}-f^{(S)}(\bar{y}) \leqslant 8 r \chi \bar{y}$. Тогда решение $y(t)$ уравнения (3.6) с начальным значением $y\left(t_{0}\right)=y_{0}$ имеет конечный предел $\lim _{t \rightarrow+\infty} y(t)=\hat{y}$.

ДокАЗАтЕльство. Изнеравенств $y_{0} \leqslant \bar{y}, \beta^{(S)}-f^{(S)}\left(y_{0}\right) \geqslant 8 r \chi y_{0}, \beta^{(S)}-f^{(S)}(\bar{y}) \leqslant$ $8 r \chi \bar{y}$ следует, что сушествует число $\overline{\bar{y}}$, удовлетворяюшее условиям $y_{0} \leqslant \overline{\bar{y}} \leqslant \bar{y}, \beta^{(S)}-$ $f^{(S)}(\overline{\bar{y}})=8 r \chi \overline{\bar{y}}$, такое, что в интервале $\left(y_{0}, \overline{\bar{y}}\right)$ нет чисел $\eta$, для которых $\beta^{(S)}-f^{(S)}(\eta)=$ $8 r \chi \eta$. Докажем, что $\lim _{t \rightarrow+\infty} y(t)=\overline{\bar{y}}$. Согласно неравенству $\beta^{(S)}-f^{(S)}\left(y_{0}\right) \geqslant 8 r \chi y_{0}$ и выбору числа $\overline{\bar{y}}$, в интервале $y_{0}<y<\overline{\bar{y}}$ выполняется неравенство

$$
-f^{(S)}(y)+\beta^{(S)}-8 r \chi y>0
$$


и решение $y(t)$ уравнения (3.6) возрастает с ростом $t$. Поэтому для любого $\epsilon>0$ сушествует значение $\overline{\bar{t}} \geqslant t_{0}$ такое, что $\overline{\bar{y}}-\epsilon \leqslant y(\overline{\bar{t}}) \leqslant \overline{\bar{y}}$. Представим функцию $f^{(S)}(y)$ в окрестности точки $y=\overline{\bar{y}}$ в виде

$$
f^{(S)}(y)=\beta^{(S)}-8 r \chi \overline{\bar{y}}+b_{\nu}(y-\overline{\bar{y}})^{\nu}+O\left(|y-\overline{\bar{y}}|^{\nu+1}\right),
$$

где $\nu$ - натуральное число, $b_{\nu}$ - константа и $\left|O\left(|y-\bar{y}|^{\nu+1}\right)\right| \leqslant$ const $|y-\overline{\bar{y}}|^{\nu+1}$. Из этого представления следует, что для достаточно малого $\epsilon>0$

$$
\int_{\overline{\bar{y}}-\epsilon}^{\bar{y}} \frac{d y}{-f^{(S)}(y)+\beta^{(S)}-8 r \chi y}=\infty .
$$

Поэтому в силу (3.6) либо для любого $t \geqslant t_{0}$ решение $y(t)$ удовлетворяет неравенству $y(t)<\overline{\bar{y}}$, либо при всех $t \geqslant t_{0} y(t) \equiv \overline{\bar{y}}$. Лемма доказана.

Лемма 3.7. Предположим, что $\beta^{(S)}-\gamma_{S}^{\prime \prime} \leqslant 8 r \chi y_{0} \leqslant \beta^{(S)}-\gamma_{S}^{\prime}, \beta^{(S)}-f^{(S)}\left(y_{0}\right) \leqslant$ 8 г $\chi y_{0}$ и существует число $\tilde{y} \leqslant y_{0}$ такое, что $\beta^{(S)}-f^{(S)}(\tilde{y}) \geqslant 8 r \chi y_{0}$. Тогда решение $y(t)$ уравнения (3.6) с начальным значением $y\left(t_{0}\right)=y_{0}$ имеет конечный предел $\lim _{t \rightarrow+\infty} y(t)=\hat{y}$.

ДокАЗАТЕЛЬСТво проводится так же, как доказательство леммы 3.6 .

Будем искать такое решение $\left(x_{\vec{n}}^{(S)}(t)\right)$ системы $(1.5)$, чтобы для любых двух векторов $\vec{n}^{\prime}=\left(n_{1}^{\prime}, \ldots, n_{r}^{\prime}\right) \in \mathbf{Z}^{r}, \vec{n}^{\prime \prime}=\left(n_{1}^{\prime \prime}, \ldots, n_{r}^{\prime \prime}\right) \in \mathbf{Z}^{r}$, удовлетворяюших равенству (3.4), выполнялось равенство (3.5). Используя определение множества $\Gamma_{\vec{n}}$ в уравнении (1.5), получим, что если $\vec{n}^{\prime} \in \Gamma_{\vec{n}}$, то функции $x_{\vec{n}^{\prime}}^{(S)}$ в уравнении $(1.5)$ удовлетворяют тождеству

$$
x_{\vec{n}^{\prime}}^{(S)}(t) \equiv-x_{\vec{n}}^{(S)}(t)
$$

и равенство (1.5) принимает вид уравнения

$$
\frac{d x_{\vec{n}}^{(S)}}{d t}=-f^{(S)}\left(x_{\vec{n}}^{(S)}\right)+\beta^{(S)}-8 r \chi x_{\vec{n}}^{(S)}
$$

относительно функции $x_{\vec{n}}^{(S)}(t)$, которое совпадает с уравнением (3.6) относительно функции $y(t)$. Так как согласно равенству (3.3) справедливо тождество $\beta^{(S)}-f^{(S)}\left(x_{\vec{n}}^{(S)}\right) \equiv$ $-\left(\beta^{(S)}-f^{(S)}\left(-x_{\vec{n}}^{(S)}\right)\right)$, то в силу (3.10) уравнение (3.11) справедливо для любого вектора $\vec{n}^{\prime} \in \Gamma_{\vec{n}}$ :

$$
\frac{d x_{\vec{n}^{\prime}}^{(S)}}{d t}=-f^{(S)}\left(x_{\vec{n}^{\prime}}^{(S)}\right)+\beta^{(S)}-8 r \chi x_{\vec{n}^{\prime}}^{(S)}
$$

Поэтому, если уравнение (3.11) справедливо для некоторого вектора $\vec{n}$, то оно будет справедливо для всех $\vec{n} \in \mathbf{Z}^{r}$, и чтобы найти решения $\left(x_{\vec{n}}^{(S)}(t)\right)$ системы (1.5), удовлетворяюшие условию (3.5), необходимо найти все решения уравнения (3.6), которые однозначно определены заданием начального значения $y_{0}=y\left(t_{0}\right)$. Теперь все утверждения теоремы 3.2 непосредственно следуют из лемм $3.4-3.7$. Теорема доказана. 
СлЕДСтвИЕ 3.3. Пусть константа $\beta^{(S)}=0$, а функция $f^{(S)}(x)$ нечетна: $f^{(S)}(x)=-f^{(S)}(-x)$. Тогда справедливы все утверждения теоремы 3.2.

ПримеР. $\beta^{(S)}=0, f^{(S)}(x)=b \sin (\omega x)$, где $b$ и $\omega-$ константы.

СлЕДСтвИЕ 3.4. Если функция $f^{(S)}(x)$ - периодическая, выполнено равенство (3.3) и справедливо неравенство $\beta^{(S)}-\gamma_{S}^{\prime \prime} \leqslant 8 r \chi x_{0}^{(S)} \leqslant \beta^{(S)}-\gamma_{S}^{\prime}$, то при любом $\vec{n} \in \mathbf{Z}^{r}$ существует конечный предел $\lim _{t \rightarrow+\infty} x_{\vec{n}}^{(S)}(t)=\hat{x}^{(S)}$.

СлЕДСтвиЕ 3.5. Если функция $f^{(S)}(x)$ периодическая, $\vec{n}^{\prime}$ и $\vec{n}^{\prime \prime}-$ вектора с иельми координатами, удовлетворяющие (3.4), и выполнено равенство (3.3), то $x_{0}^{(S)}=\left(\beta^{(S)}-\gamma_{S}^{\prime \prime}\right) /(8 r \chi), \quad x_{0}^{(S)}=\left(\beta^{(S)}-\gamma_{S}^{\prime}\right) /(8 r \chi)-$ критические значения, при прохождении через которые решения $\left(x_{\vec{n}}^{(S)}(t)\right)$ системь $(1.5)$ с начальными даннымми $x_{\vec{n}^{\prime}}^{(S)}\left(t_{0}\right)=x_{0}^{(S)}, \quad x_{\vec{n}^{\prime \prime}}^{(S)}\left(t_{0}\right)=-x_{0}^{(S)}$ принципиально меняют свое поведение в пределе $t \rightarrow+\infty$.

Благодарности. Выражаю глубокую благодарность научному центру $\mathrm{BiBoS}$ университета Bielefeld за поддержку.

\section{Список литературы}

[1] Я. Г. Синай. Теория фазовых переходов. Строгие результаты. М.: Наука, 1980.

[2] P. Bak, C. Tang, K. Wiesenfeld. Phys. Rev. Lett. 1987. V. 59. № 4. P. 381; Phys. Rev. A. 1988. V. 38. № 1. P. 364; P. Bak. How Nature Works. Berlin: Springer, 1996; D. Dhar. Phys. Rev. Lett. 1990. V. 64. P. 1613; D. Dhar, S.N. Majumdar. J. Phys. A. 1990. V. 23. P. 4333; S. N. Majumdar, D. Dhar. Physica A. 1992. V. 185. P. 129; D. Dhar, R. Ramaswamy. Phys. Rev. Lett. 1989. V. 63. P. 1659; Díaz-guilera. Europhys. Lett. 1994. V. 26. № 3. P. 177; Díaz-guilera. Phys. Rev. A. 1992. V. 45. № 12. P. 8551; Europhys. Lett. 1994. V. 26. № 3. P. 177; H. J. Jensen. Self-Organized Criticality. Emergent Complex Behavior in Physical and Biological systems. Cambridge: Cambridge Univ. Press, 1998; L. Pietronero, P. Tartaglia, Y.C. Zhang. Physica A. 1991. V. 173. P. 22; R. Cafiero, V. Loreto, L. Pietronero, A. Vespignani, S. Zapperi. Europhys. Lett. 1995. V. 129. № 2. P. 111; L. Pietronero, A. Vespignani, S. Zapperi. Phys. Rev. Lett. 1994. V. 72. P. 1690; A. Vespignani, S. Zapperi, L. Pietronero. Phys. Rev. E. 1995. V. 51. P. 1711; H. Y. Zhang. Phys. Rev. Lett. 1988. V. 63. № 5. P. 470; S. N. Coppersmith, D.S. Fisher. Threshold behavior of a driven incommensurate harmonic chain. Preprint. Princeton: Princeton University, 1988; R. Burridge, L. Knopoff. Bull. Seismol. Sos. Am. 1967. V. 57. P. 341.

[3] Итоги науки и техники. Современные проблемы математики. Фундаментальные направления. Т. 5. Ред. Р.В. Гамкрелидзе. М.: ВИНИТИ, 1986.

[4] Л. Д. Пустыльников. ТМФ. 1992. Т. 92. № 1. С. 85.

[5] Л. Д. Пустыльников. Критические явления и бифуркации решений бесконечномерных систем обыкновенных дифференциальных уравнений, возникающих в некоторых физических проблемах. Препринт № 43. М.: ИПМ РАН, 2001; Пространственно-временной хаос и теория бесконечномерных систем обыкновенных дифференциальных уравнений, возникающих в некоторых физических проблемах. Препринт № 44. М.: ИПМ РАН, 2001.

[6] V.G. Gelfreich, V.F. Lazutkin, N.V. Svanidze. Refined formula to separatrix spliting for the standard map. Warwick Preprints. № 59, 1992. 